\title{
Dynamics of the Size and Orientation Distribution of Microcracks and Evolution of Macroscopic Damage Parameters
}

\author{
Christina Papenfuss $s^{1,3, *}$, Thomas Böhme ${ }^{1}$, Heiko Herrmann ${ }^{3}$, \\ Wolfgang Muschik ${ }^{3}$, and Joseph Verhás ${ }^{2}$ \\ ${ }^{1}$ Technische Universität Berlin, Institut für Mechanik, Straße des 17. Juni, 10623 \\ Berlin, Germany \\ ${ }^{2}$ Budapest University of Technology and Economics, Department of Chemical \\ Physics, Budafoki út 8, 1521 Budapest, Hungary \\ ${ }^{3}$ Technische Universität Berlin, Institut für Theoretische Physik, Hardenbergstr. \\ 36, 10623 Berlin, Germany
}

*Corresponding author (c.papenfuss@gmx.de)

Communicated by K.-H. Hoffmann, Chemnitz, Germany

\begin{abstract}
We are dealing with damage of brittle materials caused by growth of microcracks. In our model the cracks are penny-shaped. They can only enlarge but not heal. For a single crack a Rice-Griffith growth law is assumed: There is crack growth only if tension is applied normally to the crack surface, exceeding a critical value. Our aim is to investigate the effect of crack growth on macroscopic constitutive quantities. A possible approach taking into account such an internal structure within continuum mechanics is the mesoscopic theory. A distribution of crack lengths and crack orientations within the continuum element is introduced. Macroscopic quantities are calculated as averages with the distribution function. A macroscopic measure of the progressing damage, i.e., a damage parameter, is the average crack length. For this scalar damage parameter we derive an evolution equation. Due to the unilateral growth law for the single crack, it turns out that the form of this differential equation depends explicitly on the initial crack length distribution. In order to treat biaxial loading, it is necessary to introduce a tensorial damage parameter. We define a second-order tensor damage parameter in terms of the crack length and orientation distribution function.
\end{abstract}




\section{Introduction}

Macroscopic failure of brittle materials is initiated by the propagation of microcracks. In a simple model, the microcrack is described as a flat, rotationally symmetric surface, a so-called penny-shaped crack. In addition, we make here the following simplifying assumptions:

1. The diameter of the cracks is much smaller than the linear dimension of the continuum element. Under this assumption, the cracks can be treated as an internal structure of the continuum element. The cracks are assumed small enough that there is a whole distribution of crack sizes and orientations in the volume element.

2. The cracks are fixed to the material. Therefore, their motion is coupled to the motion of representative volume elements.

3. The cracks cannot rotate independently of the material, i.e., their rotation velocity is determined by the antisymmetric part of the velocity gradient of the surrounding material and it does not depend on crack length and orientation. All cracks within a volume element move and rotate with the same velocity.

4. The number of cracks is fixed, there is no production of cracks, but very short cracks are preexisting in the virgin material.

5. The cracks cannot decrease area, but can only enlarge, meaning that cracks cannot heal.

To summarize our model, the microcrack is characterized by a unit vector $n$ representing the orientation of the surface normal and by the radius $l$ of the spherical crack surface. These parameters will be taken as the additional variables in the mesoscopic theory.

\section{Mesoscopic theory}

The mesoscopic theory has been developed in order to deal with complex materials within continuum mechanics [1]. The idea is to enlarge the domain of the field quantities by an additional variable, characterizing the internal degree of freedom connected to the internal structure of the material. In our case, the internal degrees of freedom are the different sizes $l$ and orientations $\boldsymbol{n}$ of microcracks (see also [2-4]).

Beyond the use of additional variables the mesoscopic concept introduces a statistical element, the so-called mesoscopic distribution function. In our case, this is a distribution of crack lengths and orientations in the continuum element at position $\boldsymbol{x}$ and time $t$, called here crack distribution function $(\mathrm{CDF})$. The distribution function is the probability density of finding a crack 
of length $l$ and orientation $\boldsymbol{n}$ in the continuum element. The elements are material elements, including the same material and the same cracks for all times. Macroscopic quantities are calculated from mesoscopic ones as averages over crack sizes and crack orientations.

\subsection{Mesoscopic balance equations}

Field quantities such as mass density, momentum density, angular momentum density, and energy density are defined on the mesoscopic space. For distinguishing these fields from the macroscopic ones, we add the word "mesoscopic". In addition, we introduce the crack number density $N$ as an extensive quantity. The mesoscopic crack number density $N(l, \boldsymbol{n}, \boldsymbol{x}, t)$ is the number density, counting only cracks of length $l$ and orientation $\boldsymbol{n}$. The only balance equation considered in the following is the balance of crack number.

\section{Balance of crack number}

In our model, the cracks move together with the material element. Therefore, their flux is the convective flux, having a part in position space, a part in orientation space, and a part in the length interval. There is no production and no supply of crack number. Therefore, we have for the crack number density $N$ :

$$
\frac{\partial}{\partial t} N(\cdot)+\nabla_{x} \cdot\{N(\cdot) \boldsymbol{v}(\boldsymbol{x}, t)\}+\nabla_{n} \cdot\{N(\cdot) \boldsymbol{u}(\cdot)\}+\frac{1}{l^{2}} \frac{\partial}{\partial l}\left(l^{2} i N(\cdot)\right)=0 .
$$

We have used spherical coordinates for the mesoscopic variables crack length $l \in[0, \infty]$ and crack orientation $\boldsymbol{n} \in S^{2}$ and represent the divergence with respect to the mesoscopic variables in spherical coordinates. $\nabla_{n}$ denotes the covariant derivative on the unit sphere. $v$ is the material velocity. In our model, all cracks within the continuum element move with this velocity. $\boldsymbol{u}(\cdot)=\dot{\boldsymbol{n}}$ is the orientation change velocity, which is not the same for all cracks in the continuum element. It is related to the angular velocity $\omega(\boldsymbol{x}, t)$ by the relation

$$
\boldsymbol{u}(\cdot)=\boldsymbol{\omega} \times \boldsymbol{n} .
$$

This angular velocity is the same for all cracks in the element. It is determined by the rotation of the surrounding material.

\subsection{Definition of the distribution function and equation of motion}

Because of its definition as probability density, the distribution function is the number fraction 


$$
f(l, \boldsymbol{n}, \boldsymbol{x}, t)=\frac{N(l, \boldsymbol{n}, \boldsymbol{x}, t)}{N(\boldsymbol{x}, t)},
$$

in volume elements, where the number density $N(\boldsymbol{x}, t)$ is non-zero. Here $N(\boldsymbol{x}, t)$ is the macroscopic number density of cracks of any length and orientation. As the distribution function in Eq. (3) is not well defined if $N(\boldsymbol{x}, t)=$ 0 , we define in addition that in this case $f(l, \boldsymbol{n}, \boldsymbol{x}, t)=0$. As there is no creation of cracks in our model, the distribution function will be zero for all times in these volume elements. In all other volume elements with a nonzero crack number it is normalized

$$
\int_{0}^{\infty} \int_{S^{2}} f(l, \boldsymbol{n}, \boldsymbol{x}, t) l^{2} d^{2} n d l=1
$$

With respect to crack length, it is supposed that the distribution function has a compact support, meaning that in a sample there cannot exist cracks larger than the sample size.

We obtain from the mesoscopic balance of the crack number density (1) a balance of the $\operatorname{CDF} f(l, \boldsymbol{n}, \boldsymbol{x}, t)$, by inserting its definition:

$$
\begin{aligned}
\frac{\partial}{\partial t} f(l, \boldsymbol{n}, \boldsymbol{x}, t)+\nabla_{x} \cdot(\boldsymbol{v}(\boldsymbol{x}, t) f(l, \boldsymbol{n}, \boldsymbol{x}, t)) \\
+\nabla_{n} \cdot(\boldsymbol{u} f(l, \boldsymbol{n}, \boldsymbol{x}, t))+\frac{1}{l^{2}} \frac{\partial}{\partial l}\left(l^{2} i f(l, \boldsymbol{n}, \boldsymbol{x}, t)\right) \\
=\frac{f(l, \boldsymbol{n}, \boldsymbol{x}, t)}{N(\boldsymbol{x}, t)}\left(\frac{\partial N(\boldsymbol{x}, t)}{\partial t}+\boldsymbol{v}(\boldsymbol{x}, t) \cdot \nabla_{x} N(\boldsymbol{x}, t)\right) \\
=-f(l, \boldsymbol{n}, \boldsymbol{x}, t) \nabla_{x} \cdot \boldsymbol{v}(\boldsymbol{x}, t),
\end{aligned}
$$

according to the balance of the total crack number $N(\boldsymbol{x}, t) . i$ is the length change velocity. In our model, all cracks in a volume element move with the translational velocity of the volume element $\boldsymbol{v}(\boldsymbol{x}, t)$ and rotate with a velocity independent of crack size and orientation:

$$
\boldsymbol{\omega}(\boldsymbol{x}, t)=\nabla \times \boldsymbol{v}(\boldsymbol{x}, t)
$$

given by the rotation of the axes of the material element. The orientation change velocity $\boldsymbol{u}$ is given by

$$
\boldsymbol{u}=\boldsymbol{n} \times \boldsymbol{\omega}(\boldsymbol{x}, t) .
$$


Therefore, we have (with the totally antisymmetric third-order tensor $\varepsilon$ ):

$$
\begin{aligned}
\nabla_{n} \cdot & (\boldsymbol{u} f(l, \boldsymbol{n}, \boldsymbol{x}, t))=\nabla_{n} \cdot(\boldsymbol{n} \times \boldsymbol{\omega}(\boldsymbol{x}, t) f(l, \boldsymbol{n}, \boldsymbol{x}, t)) \\
& =\nabla_{n} \cdot(\boldsymbol{\varepsilon}: \boldsymbol{n} \boldsymbol{\omega}(\boldsymbol{x}, t) f(l, \boldsymbol{n}, \boldsymbol{x}, t)) \\
& =\underbrace{(\boldsymbol{\delta}-\boldsymbol{n n}): \boldsymbol{\varepsilon}}_{=0} \cdot \boldsymbol{\omega}(\boldsymbol{x}, t) f(l, \boldsymbol{n}, \boldsymbol{x}, t)+(\boldsymbol{\varepsilon}: \boldsymbol{n} \boldsymbol{\omega}(\boldsymbol{x}, t)) \cdot \nabla_{n} f(l, \boldsymbol{n}, \boldsymbol{x}, t) \\
& =\boldsymbol{u} \cdot \nabla_{n} f(l, \boldsymbol{n}, \boldsymbol{x}, t),
\end{aligned}
$$

where it has been used that the covariant derivative of the unit vector $\boldsymbol{n}$ is the projector on the tangential plane to the unit sphere:

$$
\nabla_{n} \boldsymbol{n}=\boldsymbol{P}=\boldsymbol{\delta}-\boldsymbol{n n} .
$$

The first three terms on the left-hand side of Eq. (5) can be summarized with the abbreviation $\frac{d^{c}}{d t}$ :

$$
\begin{aligned}
& \frac{\partial}{\partial t} f(l, \boldsymbol{n}, \boldsymbol{x}, t)+\boldsymbol{v}(\boldsymbol{x}, t) \cdot \nabla_{x} f(l, \boldsymbol{n}, \boldsymbol{x}, t) \\
& \quad+\boldsymbol{u}(\boldsymbol{n}, \boldsymbol{x}, t) \cdot \nabla_{n} f(l, \boldsymbol{n}, \boldsymbol{x}, t)=\frac{d^{c} f(l, \boldsymbol{n}, \boldsymbol{x}, t)}{d t} .
\end{aligned}
$$

We end up with the equation of motion for the CDF:

$$
\begin{aligned}
& \frac{d^{c} f(l, \boldsymbol{n}, \boldsymbol{x}, t)}{d t}+\frac{1}{l^{2}} \frac{\partial}{\partial l}\left(l^{2} i f(l, \boldsymbol{n}, \boldsymbol{x}, t)\right)+\left(\nabla_{x} \cdot \boldsymbol{v}\right) f(l, \boldsymbol{n}, \boldsymbol{x}, t) \\
& =\left(\nabla_{x} \cdot \boldsymbol{v}\right) f(l, \boldsymbol{n}, \boldsymbol{x}, t)
\end{aligned}
$$

or

$$
\frac{d^{c} f(l, \boldsymbol{n}, \boldsymbol{x}, t)}{d t}+\frac{1}{l^{2}} \frac{\partial}{\partial l}\left(l^{2} i f(l, \boldsymbol{n}, \boldsymbol{x}, t)\right)=0 .
$$

This is not yet a closed differential equation for the CDF as long as no expression for the length change velocity of the crack $i$ is given.

\subsection{Single crack growth law}

For the growth velocity of a single crack under a prescribed external load, we make the following assumptions: 
1. There exists a critical crack length, depending on the load, such that only cracks exceeding this length start growing. This critical length $l_{c}$ is given by the Griffith criterion for the onset of growth [5]:

$$
l_{c}=\frac{K}{\sigma_{n}^{2}},
$$

where $K$ is a material constant, and $\sigma_{n}$ is the stress applied perpendicularly to the crack surface. It is assumed that a stress component within the crack plane does not cause crack growth. In case of an orientation distribution of cracks, the normal stress component $\sigma_{n}$ is proportional to the external load and depends on crack orientation.

2. The growth velocity is given by the Rice-Griffith dynamics, which is motivated from macroscopic thermodynamic considerations:

$$
\begin{aligned}
& i=-\alpha+\beta \sigma_{n}^{2} l \quad \text { for } l \geq l_{c}, \\
& i=0 \text { for } l<l_{c}
\end{aligned}
$$

with material coefficients $\alpha$ and $\beta$. The onset of growth starts when the right-hand side of Eq. (13) becomes positive, which determines the critical length $l_{c}$. Therefore, the material parameter $K$ in Eq. (12) is not independent of $\alpha$ and $\beta$ :

$$
K=\frac{\alpha}{\beta} .
$$

In the case of a constant time rate of the applied stress, $\sigma_{n}=v_{\sigma} t$, it results:

$$
\begin{aligned}
& i=-\alpha+\beta v_{\sigma}^{2} l t^{2} \quad \text { for } l \geq l_{c}, \\
& i=0 \text { for } l<l_{c} .
\end{aligned}
$$

$v_{\sigma}$ is the time derivative of the applied stress normal to the crack surface.

With this model for the length change velocity, we end up with the following differential equation for the distribution function:

$$
\begin{aligned}
& \frac{d^{c} f(l, \boldsymbol{n}, \boldsymbol{x}, t)}{d t}=-\frac{1}{l^{2}} \frac{\partial}{\partial l}\left(l^{2}\left(-\alpha+\beta v_{\sigma}^{2} l t^{2}\right)\right) \quad \text { for } l \geq l_{c}, \\
& \frac{d^{c} f(l, \boldsymbol{n}, \boldsymbol{x}, t)}{d t}=0 \quad \text { for } l<l_{c} .
\end{aligned}
$$



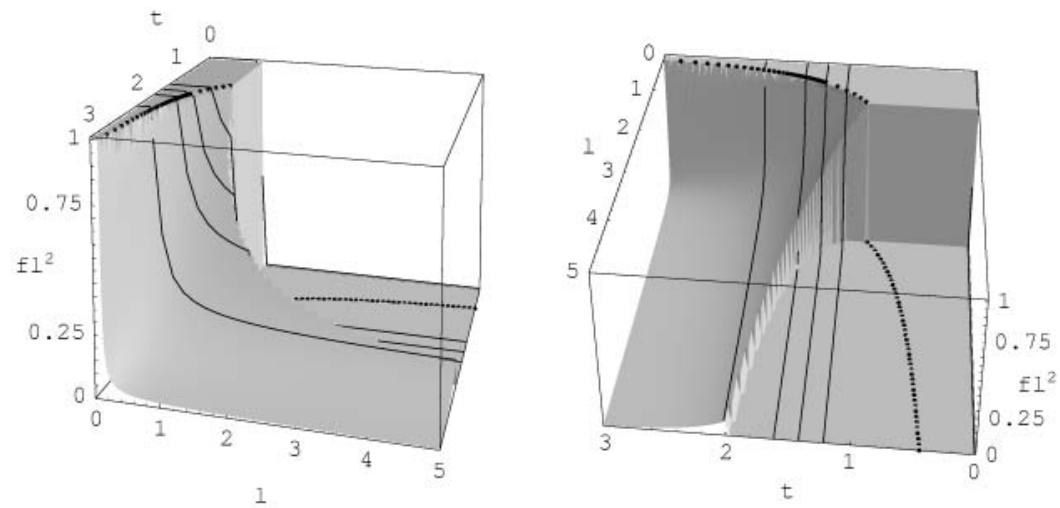

Figure 1 Evolution of the crack length distribution in time for stepwise initial length distribution with cracks present up to length 1 . Model parameters have been set equal to 1 . Both panels represent different views of the same graphic. The dotted line and its projection onto the $l-t$ plane is the Griffith condition, which is more apparent on the backward view.

In addition, we define the length distribution function as the integral of the CDF over all orientations:

$$
f(l, \boldsymbol{x}, t)=\int_{S^{2}} f(l, \boldsymbol{n}, \boldsymbol{x}, t) d^{2} n
$$

It gives the probability density of finding a crack of length $l$, of any orientation in the volume element at position $\boldsymbol{x}$ and time $t$. The differential equation for this length distribution is obtained by integrating Eq. (19) over all orientations. Solutions of the resulting differential equation are discussed in [3], and one example of a solution is shown in Figure 1.

\section{Orientational order parameters and dynamics of the orientational order}

The orientation distribution of cracks is relevant, too, because only the component of the stress vector parallel to the orientation $\boldsymbol{n}$ causes crack growth, i.e., in a sample where most crack surface normals are parallel to the direction of the applied stress, crack growth is more pronounced than in a sample where most crack normals are perpendicular to the stress direction. Macroscopic quantities that account for orientational order are the Fabric alignment tensors, which are defined as [4]:

$$
\boldsymbol{a}^{(k)}=\int_{0}^{\infty} \int_{S^{2}} f(l, \boldsymbol{n}, \boldsymbol{x}, t) \underbrace{\stackrel{\boldsymbol{n} \ldots \boldsymbol{n}}{n}}_{k \text { times }} d^{2} n l^{2} d l .
$$


They are tensorial moments of the orientation distribution, i.e., tensors of successive order. In what follows $\ldots$ denotes the symmetric irreducible part of a tensor, i.e. the contraction over any pair of indices of it vanishes. The odd order tensors $\boldsymbol{a}^{(k)}$ vanish due to the symmetry $f(l, \boldsymbol{n}, \boldsymbol{x}, t)=f(l,-\boldsymbol{n}, \boldsymbol{x}, t)$, which expresses the fact that surface normals $\boldsymbol{n}$ and $-\boldsymbol{n}$ cannot be distinguished. Therefore, the first nonvanishing anisotropic moment is the secondorder Fabric alignment tensor, which is the most important one. These Fabric alignment tensors are the macroscopic variables representing the orientational order present at the mesoscopic level. They, especially the second-order tensor, can be taken as variables in macroscopic constitutive theory. The differential equation for them can be derived from the differential equation for the CDF, Eq. (5). This is shown here in the case of the second-order tensor $\boldsymbol{a}^{(2)}=\boldsymbol{a}$ :

$$
\begin{aligned}
\frac{d \boldsymbol{a}}{d t} & =\frac{d}{d t} \int_{0}^{\infty} \int_{S^{2}} f(l, \boldsymbol{n}, \boldsymbol{x}, t) \overline{\boldsymbol{n n}} d^{2} n l^{2} d l \\
& =\int_{0}^{\infty} \int_{S^{2}}\left(\frac{\partial}{\partial t}+\boldsymbol{v}(\boldsymbol{x}, t) \cdot \nabla_{x}\right) f(l, \boldsymbol{n}, \boldsymbol{x}, t) \overline{\boldsymbol{n n}} d^{2} n l^{2} d l \\
& =\int_{0}^{\infty} \int_{S^{2}}\left(-\boldsymbol{u}(\boldsymbol{n}, \boldsymbol{x}, t) \cdot \nabla_{n} f(l, \boldsymbol{n}, \boldsymbol{x}, t)-\frac{1}{l^{2}} \frac{\partial}{\partial l}\left(l^{2} l f(l, \boldsymbol{n}, \boldsymbol{x}, t)\right)\right) \overline{\boldsymbol{n n}} d^{2} n l^{2} d l \\
& =-\int_{S^{2}} \boldsymbol{u}(\boldsymbol{n}, \boldsymbol{x}, t) \cdot \nabla_{n}(f(\boldsymbol{n}, \boldsymbol{x}, t)) \overline{\boldsymbol{n n}} d^{2} n \\
& =-\int_{S^{2}}(\boldsymbol{\omega} \times \boldsymbol{n}) \cdot \nabla_{n}(f(\boldsymbol{n}, \boldsymbol{x}, t)) \overline{\boldsymbol{n n}} d^{2} n \\
& =\int_{S^{2}}(\boldsymbol{\omega} \cdot \boldsymbol{\varepsilon}: \boldsymbol{P}+\boldsymbol{\omega} \times \boldsymbol{n n}-\boldsymbol{n n} \times \boldsymbol{\omega}) f(\boldsymbol{n}, \boldsymbol{x}, t) d^{2} n \\
& =\int_{S^{2}}(0+\boldsymbol{\omega} \times \boldsymbol{n n}-\boldsymbol{n n} \times \boldsymbol{\omega}) f(\boldsymbol{n}, \boldsymbol{x}, t) d^{2} n \\
& =\boldsymbol{\omega} \times \boldsymbol{a}-\boldsymbol{a} \times \boldsymbol{\omega},
\end{aligned}
$$

i.e., the co-rotational time derivative of the second-order alignment tensor vanishes. This is due to the fact that for an observer moving and rotating with the material element, the orientational order of the cracks does not change, because the cracks are moving and rotating with the same velocity. Therefore, for the co-moving observer the Fabric alignment tensors are constant.

\section{Average crack length as a scalar damage parameter}

The damage parameter is introduced as a macroscopic quantity growing with progressive damage in such a way that it should be possible to relate 
the change of material properties to the growth of the damage parameter. One possibility is to define a scalar damage parameter as the average crack length [3]:

$$
\begin{aligned}
D(\boldsymbol{x}, t)=\langle l\rangle & =\int_{0}^{\infty} \int_{S^{2}} l f(l, \boldsymbol{n}, \boldsymbol{x}, t) d^{2} n l^{2} d l \\
& =\int_{0}^{\infty} \int_{S^{2}} f(l, \boldsymbol{n}, \boldsymbol{x}, t) d^{2} n l^{3} d l .
\end{aligned}
$$

This can be expressed in terms of the length distribution function:

$$
D(\boldsymbol{x}, t)=\int_{0}^{\infty} f(l, \boldsymbol{x}, t) l^{3} d l
$$

In order to account for the anisotropic nature of damage, a second-order damage parameter will be appropriate. A definition of such a tensorial damage parameter, which is closely related to the inelastic strain in a damaged sample (as we will show in a forthcoming paper), is:

$$
\boldsymbol{D}=\int_{0}^{\infty} \int_{S^{2}} f(l, \boldsymbol{n}, \boldsymbol{x}, t) \ln \boldsymbol{n} d^{2} n l^{2} d l
$$

\subsection{Dynamics of the scalar damage parameter}

With the Rice-Griffith dynamics in the special case of unilateral stress with a constant stress rate, we derive for the damage parameter the following differential equation:

$$
\begin{aligned}
\dot{D}(\boldsymbol{x}, t)= & \int_{0}^{l_{c}(t)} \underbrace{\frac{d}{d t}(f(l, \boldsymbol{x}, t)) l^{3}}_{=0 \text { because } l<l_{c}} d l \\
& +\frac{d l_{c}(t)}{d t} l_{c}^{3} f\left(l_{c}\right)+\int_{l_{c}(t)}^{\infty} \frac{d}{d t}(f(l, \boldsymbol{x}, t)) l^{3} d l-\frac{d l_{c}(t)}{d t} l_{c}^{3} f\left(l_{c}\right) \\
= & \int_{l_{c}(t)}^{\infty} \frac{d}{d t}(f(l, \boldsymbol{x}, t)) l^{3} d l=\int_{l_{c}(t)}^{\infty}-\frac{1}{l^{2}} \frac{\partial}{\partial l}\left(l^{2} i f(l, \boldsymbol{x}, t)\right) l^{3} d l \\
= & \int_{l_{c}(t)}^{\infty} l^{2} i f(l, \boldsymbol{x}, t) d l-\left.\left(l l^{2} i f(l, \boldsymbol{x}, t)\right)\right|_{l_{c}(t)} ^{\infty} \\
= & \int_{l_{c}(t)}^{\infty} l^{2} i_{f} f(l, \boldsymbol{x}, t) d l+l_{c}^{3} \underbrace{\dot{l}_{(}\left(l_{c}\right)}_{=0} f\left(l_{c}, \boldsymbol{x}, t\right),
\end{aligned}
$$


with

$$
\begin{aligned}
l_{c}(t)= & \frac{\alpha^{\prime}}{\beta^{\prime} v_{\sigma}^{2} t^{2}} \quad \text { and } \quad i=-\alpha^{\prime}+\beta^{\prime} v_{\sigma}^{2} l t^{2}, \\
\dot{D}(t)= & \int_{\alpha^{\prime} / \beta^{\prime} v_{\sigma}^{2} t^{2}}^{\infty} l^{2}\left(-\alpha^{\prime}+\beta^{\prime} v_{\sigma}^{2} l t^{2}\right) f(l, \boldsymbol{x}, t) d l \\
= & \int_{0}^{\infty} l^{2}\left(-\alpha^{\prime}+\beta^{\prime} v_{\sigma}^{2} l t^{2}\right) f(l, \boldsymbol{x}, t) d l \\
& -\int_{0}^{\alpha^{\prime} / \beta^{\prime} v_{\sigma}^{2} t^{2}} l^{2}\left(-\alpha^{\prime}+\beta^{\prime} v_{\sigma}^{2} l t^{2}\right) f(l, \boldsymbol{x}, t) d l \\
= & -\alpha^{\prime}+\beta^{\prime} v_{\sigma}^{2} t^{2} D(t) \\
& +\int_{0}^{\alpha^{\prime} / \beta^{\prime} v_{\sigma}^{2} t^{2}} l^{2}\left(\alpha^{\prime}-\beta^{\prime} v_{\sigma}^{2} l t^{2}\right) f(l, \boldsymbol{x}, t=0) d l .
\end{aligned}
$$

The differential equation for the damage parameter, derived here from the mesoscopic theory, depends explicitly on the initial distribution function, i.e., the equations of motion are different for different kinds of initial crack length distribution functions. Macroscopically, this initial length distribution dependence can be interpreted as a constitutive quantity. The differential equation can be written explicitly, knowing the initial distribution, but without solving the dynamics for the distribution function. The equation of motion can be written in such a form that the distribution at the actual time $t$ does not occur. This means that for the average crack length as the damage parameter we can solve the differential equation (28) on the macroscopic level without any need to solve a differential equation on the higher dimensional mesoscopic space. This property is very convenient from a numerical point of view, when damage evolution should be considered together with balance of momentum and balance of energy for macroscopic structures. This likely property is lost for modifications of the single crack dynamics.

\subsection{Dynamics for special initial conditions}

4.2.1. Stepwise initial condition We consider an initial crack distribution, which gives a constant probability density for crack sizes smaller than a maximal size $l_{f}$ and zero probability for larger sizes:

$$
f(l, 0)= \begin{cases}\frac{l^{-2}}{l_{f}} & \text { if } l<l_{f} \\ 0 & \text { otherwise }\end{cases}
$$


For the dynamics of the damage parameter we have to distinguish two different cases:

1. Case $l_{f}<l_{c}$ (beginning of damage):

$$
\dot{D}(t)=\int_{l_{c}(t)}^{\infty} l^{2} l f(l, \boldsymbol{x}, t) d l=0 .
$$

2. Case $l_{f}>l_{c}$ (later stage, i.e., larger stress):

$$
\begin{aligned}
\dot{D}(t) & =-\alpha^{\prime}+\beta^{\prime} v_{\sigma}^{2} t^{2} D(t)+\int_{0}^{\alpha^{\prime} / \beta^{\prime} v_{\sigma}^{2} t^{2}} l^{2}\left(\alpha^{\prime}-\beta^{\prime} v_{\sigma}^{2} l t^{2}\right) \frac{l^{-2}}{l_{f}} d l \\
& =-\alpha^{\prime}+\beta^{\prime} v_{\sigma}^{2} t^{2} D(t)+\frac{\alpha^{\prime}}{l_{f}} \frac{\alpha^{\prime}}{\beta^{\prime} v_{\sigma}^{2} t^{2}}-\frac{\beta^{\prime}}{l_{f}} v_{\sigma}^{2} t^{2} \frac{1}{2}\left(\frac{\alpha^{\prime}}{\beta^{\prime} v_{\sigma}^{2} t^{2}}\right)^{2} \\
& =-\alpha^{\prime}+\beta^{\prime} v_{\sigma}^{2} t^{2} D(t)+\frac{\alpha^{\prime 2}}{2 l_{f} \beta^{\prime} v_{\sigma}^{2} t^{2}} .
\end{aligned}
$$

The solution of this differential equation can be given analytically in terms of the error-function $\Gamma$.

4.2.2. Exponential initial condition An initial crack length distribution function decreasing exponentially with crack length is considered

$$
f(l, 0)=l^{-2} \frac{e^{-l / \delta}}{\delta} .
$$

In this case, the differential equation for the damage parameter reads:

$$
\begin{aligned}
\dot{D}(t) & =-\alpha^{\prime}+\beta^{\prime} v_{\sigma}^{2} t^{2} D(t)+\int_{0}^{\alpha^{\prime} / \beta^{\prime} v_{\sigma}^{2} t^{2}} l^{2}\left(\alpha^{\prime}-\beta^{\prime} v_{\sigma}^{2} l t^{2}\right) \frac{1}{l^{2}} \frac{e^{-l / \delta}}{\delta} d l \\
& =-\alpha^{\prime}+\beta^{\prime} v_{\sigma}^{2} t^{2} D(t)+\left.\left[-\alpha^{\prime} e^{-l / \delta}+\frac{\beta^{\prime} v_{\sigma}^{2} t^{2}}{\delta}\left(\delta^{2}+\delta l\right) e^{-l / \delta}\right]\right|_{0} ^{\alpha^{\prime} / \beta^{\prime} v_{\sigma}^{2} t^{2}} \\
& =\beta^{\prime} v_{\sigma}^{2} t^{2}\left(D(t)+\delta\left(e^{-\alpha^{\prime} / \beta^{\prime} v_{\sigma}^{2} t^{2} \delta}-1\right)\right)
\end{aligned}
$$

Solutions of the differential equations for the damage parameter in the cases of stepwise and exponential initial conditions are shown in Figure 2. The pronounced increase of damage starts at somewhat earlier times for the exponential initial condition. This is reasonable, because in this case there are cracks from the beginning that exceed the critical length and start growing. By contrast, in the case of the stepwise initial condition for a small applied load there are no cracks fulfilling the Griffith criterion for the onset of growth. 


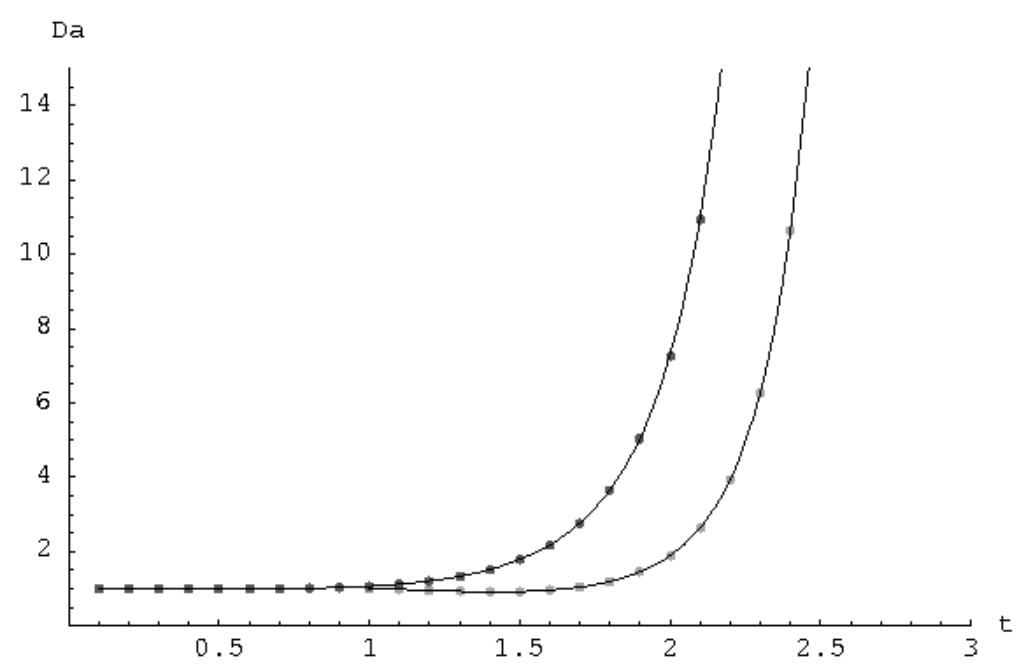

Figure 2 Time evolution of damage for stepwise (gray dots) and exponential (black dots) initial conditions. The model parameters $\alpha^{\prime}, \beta^{\prime}, v_{\sigma}, l_{c}$, and $\delta$ have been set equal to 1 .

The solution of the differential equation for $D$ is shown together with the result for the damage parameter calculated directly from the distribution function by numerical integration (the dots in the figure), i.e., calculating $D=\int_{0}^{\infty} f l^{3} d l$. The figure shows that the macroscopic evolution equation for the damage parameter gives exactly the same result on the time-depending damage parameter as the mesoscopic equation for the distribution function, i.e. there is no need to solve the differential equation for the distribution function on the higher dimensional space $\mathbb{R}_{x}^{3} \times \mathbb{R}_{t} \times[0, \infty] \times S^{2}$.

\section{Conclusions}

In the mesoscopic description we have introduced mesoscopic fields, defined on an enlarged space including crack size and orientation. Averages over crack sizes and orientations, i.e., macroscopic quantities, are calculated with a distribution function $f$. The differential equation for this distribution function was derived from the mesoscopic balance equations and crack growth law for the single crack.

A scalar damage parameter, the average crack length, has been defined, and the equation of motion for the damage parameter in the case of uniaxial loading with stress growing linearly with time, has been derived. It turns out that the differential equation depends explicitly on the initial crack length distribution. Solutions of the differential equation have been shown for stepwise and exponential initial crack length distribution. 
Other definitions of scalar damage parameters and the corresponding evolution laws will be discussed in a future paper.

The Rice-Griffith dynamics, applied here to describe the growth of a single micro-crack, has the advantage that it leads to a simple closed form of differential equation for the scalar damage parameter. However, this growth law leads to infinite growth velocities of a single crack with time increasing $\left(\lim _{t \rightarrow \infty} \dot{l}=\infty\right)$. In a future work, a modified growth law avoiding such unphysical infinite growth velocities will be introduced into the mesoscopic theory.

Another effect, not described by our simple Rice-Griffith dynamics, is the relatively slow and stable crack growth substantially below the Griffith critical length observed in some materials, like rocks. Such subcritical crack growth can be accounted for by a growth law with a probability of crack growth being proportional to an exponential function of an activation energy and the stress intensity factor (see for instance [6], page 176).

Finally, the presence of another crack at a finite distance leads to a modification of the effective applied stress on the considered crack, depending on crack distance and relative orientation. This problem has been considered for various regular distributions of cracks (see e.g., [7]). Accounting for this effect in the mesoscopic theory would lead to a single crack growth that depends on the orientation distribution of cracks. This would result in general in an anisotropic growth law.

The damage evolution equation has been derived here from the mesoscopic background independently of macroscopic thermodynamics. A thermodynamic theory of damage, including the interpretation of failure as loss of thermodynamic stability, can be found in [8]. For a comparison to experimental results, see [9].

\section{Acknowledgements}

We thank the Deutsche Akademische Austauschdienst (DAAD, U/2607) and the Deutsche Forschungsgemeinschaft (DFG) for sponsoring the cooperation between both the Departments of Physics and Chemical Physics of the Technical University of Berlin and the Budapest University of Technology and Economics. This research was supported by OTKA (T034715 and T034603), and by the VISHAY Company, 95100 Selb, Germany.

\section{References}

[1] Papenfuss, C., Theory of liquid crystals as an example of mesoscopic continuum mechanics, Comput. Mater. Sci., 19 (2000), 45-52. 
[2] Ván, P., Papenfuss, C., Muschik, W., Mesoscopic dynamics of microcracks, Phys. Rev. E, 62 (2000), 6206-6215.

[3] Ván, P., Papenfuss, C., Muschik, W., Griffith cracks in the mesoscopic microcrack theory, J. Phys. A, 37 (2004), 5315-5328.

[4] Papenfuss, C., Ván, P., Muschik, W., Mesoscopic theory of microcracks, Arch. Mech., 55 (2003), 481-499.

[5] Griffith, A.A., The theory of rupture, Trans. First Intl. Cong. Appl. Mech. Delft, 1924.

[6] Atkinson, B.K., Fracture Mechanics of Rock, Academic Press, London, 1987.

[7] Nemat, S., Hori, M., Micromechanics: Overall Properties of Heterogeneous Materials, North-Holland, Amsterdam, 1993.

[8] Ván, P., Internal thermodynamic variables and the failure of microcracked materials, J. Non-Equilib. Thermodyn., 26 (2001), 167-189.

[9] Ván, P., Vásárhelyi, B., Second law of thermodynamics and the failure of rock materials, in: Rock Mechanics in the National Interest V1, Eds., J.P. Tinucci, D. Elsworth, K.A. Heasley, pp. 767-773, Balkema, Lisse, 2001.

Paper received: 2006-01-02

Paper accepted: 2006-08-07 